H. Lee

Nagoya Math. J.

Vol. 188 (2007), 31-57

\title{
NAKAJIMA MONOMIALS AND CRYSTALS FOR SPECIAL LINEAR LIE ALGEBRAS
}

\author{
HYEONMI LEE
}

\begin{abstract}
Nakajima introduced a certain set of monomials realizing the irreducible highest weight crystals $\mathcal{B}(\lambda)$. The monomial set can be extended so that it contains crystal $\mathcal{B}(\infty)$ in addition to $\mathcal{B}(\lambda)$. We present explicit descriptions of the crystals $\mathcal{B}(\infty)$ and $\mathcal{B}(\lambda)$ over special linear Lie algebras in the language of extended Nakajima monomials. There is a natural correspondence between the monomial description and Young tableau realization, which is another realization of crystals $\mathcal{B}(\infty)$ and $\mathcal{B}(\lambda)$.
\end{abstract}

\section{$\S 1$. Introduction}

The theory of Nakajima monomials is a combinatorial scheme for realizing crystal bases of quantum groups. Nakajima introduced a certain set of monomials realizing the irreducible highest weight crystals in [16]. Kashiwara and Nakajima independently defined a crystal structure on the set of Nakajima monomials and also gave a realization of irreducible highest weight crystal $\mathcal{B}(\lambda)$ in terms of Nakajima monomials, as the connected component of the monomial set containing a maximal vector of dominant integral weight $\lambda[9],[17]$. This has lead to the belief that it should be possible to give a similar realization for $\mathcal{B}(\infty)$, which is the crystal base of the negative part $U_{q}^{-}(\mathfrak{g})$ of a quantum group over symmetrizable Kac-Moody algebra $\mathfrak{g}$, also.

Much effort has been made to give realization of $\mathcal{B}(\infty)$ over various Kac-Moody algebras. In addition to these works, in our recent works [3], [14], we gave new realization of $\mathcal{B}(\infty)$ for the finite simple Lie algebras, in terms of Young tableaux.

Starting from the realization theorem of Kashiwara and Nakajima [9], [17], we can argue that it is not possible to find the crystal $\mathcal{B}(\infty)$ within the set of Nakajima monomials with their given crystal structure. Hence,

Received June 9, 2005.

Revised December 27, 2005, September 25, 2006.

2000 Mathematics Subject Classification: Primary 17B37, 20 G05.

This work was supported in part by KOSEF Grant R01-2003-000-10012-0. 
in our work [13], we constructed the set of extended Nakajima monomials and developed a crystal structure on it, to describe $\mathcal{B}(\infty)$ in the monomial language. Actually, the set of Nakajima monomials can be embedded as a subcrystal in this set of extended Nakajima monomials. Thus, the monomial theory developed for irreducible highest weight crystal can easily be transferred to that on the extended monomial set.

As the first contribution of our present paper, we introduce explicit descriptions of the crystal $\mathcal{B}(\infty)$, in terms of extended Nakajima monomials. We restrict ourselves to special Linear Lie algebras. The extended Nakajima monomial description is obtained by relating it to the Young tableau realization [3], [14].

The second contribution of this paper is to give an explicit description of the irreducible highest weight crystal $\mathcal{B}(\lambda)$ for any dominant integral weight $\lambda$, in monomial language, for $A_{n}$ case. Another monomial description of $\mathcal{B}(\lambda)$ for this type may be found in [6], but unlike this work, there is an immediate correspondence between our description and the Young tableau realization of Kashiwara and Nakashima [10].

Our paper is organized as follows. We first review the notion of extended Nakajima monomials and the crystal structure given on the set of such monomials. Also, we cite Young tableau expression of crystal $\mathcal{B}(\infty)$ which play a crucial role in our work. We then proceed to give monomial descriptions of $\mathcal{B}(\infty)$ and $\mathcal{B}(\lambda)$. In the process of obtaining these results, we give new expressions for the Kashiwara operators acting on a certain extended Nakajima monomials, more appropriate for the situation in hand.

One strong point of the present work is that since our description has a natural correspondence with the Young tableau description, the Young tableau theory developed for $\mathcal{B}(\infty)$ and $\mathcal{B}(\lambda)$ can easily be transferred to that on the monomial set.

In closing the introduction, we remark that recently the present work has been extended to explicit monomial descriptions of $\mathcal{B}(\infty)$ for all other classical finite types and $G_{2}$ type by the author [15].

Acknowledgments. The author thanks Professors Satoshi Naito and Yoshihisa Saito for helpful information and valuable suggestions, and the referees for their helpful comments.

\section{§2. Extended Nakajima monomials and Young tableaux}

In this section, we introduce notation and cite facts that are crucial for

our work. Please refer to the references cited in the introduction or books 
on quantum groups [2], [4] for the basic concepts on quantum groups and crystal bases.

Let us first fix the basic notation.

- $I=\{1, \ldots, n\}$ : index set.

- $A=\left(a_{i j}\right)_{i, j \in I}$ : Cartan matrix of type $A_{n}$.

- $P^{\vee}=\bigoplus_{i \in I} \mathbf{Z} h_{i}:$ dual weight lattice.

- $P=\left\{\lambda \in \mathfrak{h}^{*} \mid \lambda\left(P^{\vee}\right) \subset \mathbf{Z}\right\}$ : weight lattice, where $\mathfrak{h}=\mathbf{Q} \otimes \mathbf{Z} P^{\vee}$.

- $P^{+}=\left\{\lambda \in P \mid \lambda\left(h_{i}\right) \geq 0\right.$ for all $\left.i \in I\right\}$ : the set of dominant integral weights.

- $\Pi^{\vee}=\left\{h_{i} \mid i \in I\right\}$ : the set of simple coroots.

- $\Pi=\left\{\alpha_{i} \mid i \in I\right\}$ : the set of simple roots.

- $U_{q}\left(A_{n}\right)$ : quantum group associated with the Cartan datum $\left(A, \Pi, \Pi^{\vee}\right.$, $\left.P, P^{\vee}\right)$.

- $U_{q}^{-}\left(A_{n}\right)$ : subalgebra of $U_{q}\left(A_{n}\right)$ generated by $f_{i}(i \in I)$.

- $\mathcal{B}(\lambda)$ : irreducible highest weight crystal of highest weight $\lambda$.

- $\mathcal{B}(\infty)$ : crystal base of $U_{q}^{-}\left(A_{n}\right)$.

Throughout this paper, a $U_{q}\left(A_{n}\right)$-crystal will refer to a (abstract) crystal associated with the Cartan datum $\left(A, \Pi, \Pi^{\vee}, P, P^{\vee}\right)$. The crystal base $\mathcal{B}(\infty)$ of $U_{q}^{-}\left(A_{n}\right)$ is a $U_{q}\left(A_{n}\right)$-crystal.

\subsection{Nakajima monomials}

We now recall the set of monomials discovered by Nakajima and its crystal structure and also recall their extension introduced in [13]. Both of these sets were defined for all symmetrizable Kac-Moody algebras, but we shall restrict ourselves to the $A_{n}$ case in this paper. Our exposition of the crystal structure on Nakajima monomials follows that of Kashiwara [9].

We denote by $\mathcal{M}$ the set of Nakajima monomials in the variables $Y_{i}(m)$ $(i \in I, m \in \mathbf{Z})$. That is

$$
\mathcal{M}=\left\{\begin{array}{l|l}
\prod_{(i, m) \in I \times \mathbf{Z}} Y_{i}(m)^{y_{i}(m)} & \begin{array}{l}
y_{i}(m) \in \mathbf{Z} \text { vanishes except at } \\
\text { finitely many }(i, m)
\end{array}
\end{array}\right\} .
$$

Fix any set of integers $c=\left(c_{i j}\right)_{i \neq j \in I}$ such that $c_{i j}+c_{j i}=1$, and set

$$
A_{i}(m)=Y_{i}(m) Y_{i}(m+1) \prod_{j \neq i} Y_{j}\left(m+c_{j i}\right)^{\left\langle h_{j}, \alpha_{i}\right\rangle} .
$$


The crystal structure on $\mathcal{M}$ is defined as follows. For every monomial $M=\prod_{(i, m) \in I \times \mathbf{Z}} Y_{i}(m)^{y_{i}(m)} \in \mathcal{M}$, we set

$$
\begin{aligned}
\operatorname{wt}(M) & =\sum_{i}\left(\sum_{m} y_{i}(m)\right) \Lambda_{i}, \\
\varphi_{i}(M) & =\max \left\{\sum_{k \leq m} y_{i}(k) \mid m \in \mathbf{Z}\right\}, \\
\varepsilon_{i}(M) & =\max \left\{-\sum_{k>m} y_{i}(k) \mid m \in \mathbf{Z}\right\} .
\end{aligned}
$$

We define

$$
\begin{aligned}
& \tilde{f}_{i}(M)= \begin{cases}0 & \text { if } \varphi_{i}(M)=0, \\
A_{i}\left(m_{f}\right)^{-1} M & \text { if } \varphi_{i}(M)>0,\end{cases} \\
& \tilde{e}_{i}(M)= \begin{cases}0 & \text { if } \varepsilon_{i}(M)=0, \\
A_{i}\left(m_{e}\right) M & \text { if } \varepsilon_{i}(M)>0 .\end{cases}
\end{aligned}
$$

Here

$$
\begin{aligned}
& m_{f}=\min \left\{m \mid \varphi_{i}(M)=\sum_{k \leq m} y_{i}(k)\right\}, \\
& m_{e}=\max \left\{m \mid \varepsilon_{i}(M)=-\sum_{k>m} y_{i}(k)\right\} .
\end{aligned}
$$

These Kashiwara operators, together with the maps $\varphi_{i}, \varepsilon_{i}(i \in I)$, wt, define a crystal structure on the set $\mathcal{M}[9]$. We denote by $\mathcal{M}_{c}$ the set $\mathcal{M}$ subject to the crystal structure depending on the set $c$, as given above.

The following is a realization theorem for irreducible highest weight crystal given by Kashiwara and Nakajima.

Theorem 2.1. ([9]) For a maximal vector $M \in \mathcal{M}_{c}$, the connected component of $\mathcal{M}_{c}$ containing $M$ is isomorphic to $\mathcal{B}(\operatorname{wt}(M))$.

Extended Nakajima monomials and the crystal structure on the set of such elements was introduced in [13].

Let $\mathcal{M}^{\mathcal{E}}$ be a certain set of formal monomials in the variables $Y_{i}(m)^{(0,1)}$ and $Y_{i}(m)^{(1,0)}(i \in I, m \in \mathbf{Z})$ given by

$$
\mathcal{M}^{\mathcal{E}}=\left\{\begin{array}{l|l}
\prod_{(i, m) \in I \times \mathbf{Z}} Y_{i}(m)^{y_{i}(m)} & \begin{array}{l}
y_{i}(m)=\left(y_{i}^{0}(m), y_{i}^{1}(m)\right) \in \mathbf{Z} \times \mathbf{Z} \\
\text { vanishes except at finitely many }(i, m)
\end{array}
\end{array} .\right.
$$


The product of monomials $Y_{i}(m)^{(u, v)}$ and $Y_{i}(m)^{\left(u^{\prime}, v^{\prime}\right)}$ are set to $Y_{i}(m)^{\left(u+u^{\prime}, v+v^{\prime}\right)}$, for $(u, v),\left(u^{\prime}, v^{\prime}\right) \in \mathbf{Z} \times \mathbf{Z}$. We give the lexicographic order to the set $\mathbf{Z} \times \mathbf{Z}$ of variable exponents.

Fix any set of integers $c=\left(c_{i j}\right)_{i \neq j \in I}$ such that $c_{i j}+c_{j i}=1$, and set

$$
A_{i}(m)^{ \pm 1}=Y_{i}(m)^{(0, \pm 1)} Y_{i}(m+1)^{(0, \pm 1)} \prod_{j \neq i} Y_{j}\left(m+c_{j i}\right)^{\left(0, \pm\left\langle h_{j}, \alpha_{i}\right\rangle\right)} .
$$

The crystal structure on $\mathcal{M}^{\mathcal{E}}$ is defined as follows. For each monomial $M=\prod_{(i, m) \in I \times \mathbf{Z}} Y_{i}(m)^{y_{i}(m)} \in \mathcal{M}^{\mathcal{E}}$, we set

$$
\begin{aligned}
\tilde{\mathrm{wt}}(M) & =\sum_{i}\left(\sum_{m} y_{i}(m)\right) \Lambda_{i}=\sum_{i}\left(\sum_{m}\left(y_{i}^{0}(m), y_{i}^{1}(m)\right)\right) \Lambda_{i}, \\
\tilde{\varphi}_{i}(M) & =\max \left\{\sum_{k \leq m} y_{i}(k) \mid m \in \mathbf{Z}\right\}, \\
\tilde{\varepsilon}_{i}(M) & =\max \left\{-\sum_{k>m} y_{i}(k) \mid m \in \mathbf{Z}\right\} .
\end{aligned}
$$

Notice that the coefficients of $\tilde{\mathrm{wt}}(M)$ are pairs of integers. In this setting, we have $\tilde{\varphi}_{i}(M) \geq(0,0), \tilde{\varepsilon}_{i}(M) \geq(0,0)$, and $\tilde{\mathrm{wt}}(M)=\sum_{i}\left(\tilde{\varphi}_{i}(M)-\tilde{\varepsilon}_{i}(M)\right) \Lambda_{i}$. Set

$$
\begin{aligned}
\operatorname{wt}(M) & =\sum_{i}\left(\sum_{m} y_{i}^{1}(m)\right) \Lambda_{i}, \\
\varphi_{i}(M) & =\sum_{k \leq m} y_{i}^{1}(k) \quad \text { where } \tilde{\varphi}_{i}(M)=\sum_{k \leq m}\left(y_{i}^{0}(k), y_{i}^{1}(k)\right), \\
\varepsilon_{i}(M) & =-\sum_{k>m} y_{i}^{1}(k) \quad \text { where } \tilde{\varepsilon}_{i}(M)=-\sum_{k>m}\left(y_{i}^{0}(k), y_{i}^{1}(k)\right) .
\end{aligned}
$$

Then we trivially have $\operatorname{wt}(M)=\sum_{i}\left(\varphi_{i}(M)-\varepsilon_{i}(M)\right) \Lambda_{i}$. From the above definition, $Y_{i}(m)^{(0,1)}$ has the weight $\Lambda_{i}$, and so $A_{i}(m)$ has the weight $\alpha_{i}$. We define the action of Kashiwara operators by

$$
\begin{aligned}
& \tilde{f}_{i}(M)= \begin{cases}0 & \text { if } \tilde{\varphi}_{i}(M)=(0,0), \\
A_{i}\left(m_{f}\right)^{-1} M & \text { if } \tilde{\varphi}_{i}(M)>(0,0),\end{cases} \\
& \tilde{e}_{i}(M)= \begin{cases}0 & \text { if } \tilde{\varepsilon}_{i}(M)=(0,0), \\
A_{i}\left(m_{e}\right) M & \text { if } \tilde{\varepsilon}_{i}(M)>(0,0) .\end{cases}
\end{aligned}
$$


Here,

$$
\begin{aligned}
& m_{f}=\min \left\{m \mid \tilde{\varphi}_{i}(M)=\sum_{k \leq m} y_{i}(k)\right\}, \\
& m_{e}=\max \left\{m \mid \tilde{\varepsilon}_{i}(M)=-\sum_{k>m} y_{i}(k)\right\} .
\end{aligned}
$$

Note that $y_{i}\left(m_{f}\right)>(0,0), y_{i}\left(m_{f}+1\right) \leq(0,0), y_{i}\left(m_{e}+1\right)<(0,0)$, and $y_{i}\left(m_{e}\right) \geq(0,0)$.

For any fixed set of integers $c=\left(c_{i j}\right)_{i \neq j \in I}$ such that $c_{i j}+c_{j i}=1$, the Kashiwara operators defined in (2.11) and (2.12), together with the maps $\varphi_{i}, \varepsilon_{i}(i \in I)$, and wt of (2.8) to (2.10), define a crystal structure on the set $\mathcal{M}^{\mathcal{E}}$ [13]. We refer to an element of the set $\mathcal{M}^{\mathcal{E}}$ as an extended Nakajima monomial and denote by $\mathcal{M}_{c}^{\mathcal{E}}$ the set $\mathcal{M}^{\mathcal{E}}$ subject to the crystal structure depending on the set $c$, as given above.

Remark 2.2. Now, we may give many different crystal structures to the set of extended Nakajima monomials through the choice of the set $c$. For special linear Lie algebras, all the different crystals induced from the set of extended Nakajima monomials through different choices of the set $c$, are isomorphic (see [9] or Proposition 3.2 of [13]).

Consider the set $\dot{\mathcal{M}}_{c}^{\mathcal{E}}$ of monomials $\prod_{(i, m) \in I \times \mathbf{Z}} Y_{i}(m)^{\left(y_{i}^{0}(m), y_{i}^{1}(m)\right)} \in \mathcal{M}_{c}^{\mathcal{E}}$ with $y_{i}^{0}(m)=0$ for all $(i, m)$. The set $\dot{\mathcal{M}}_{c}^{\mathcal{E}}$ is a subcrystal of $\mathcal{M}_{c}^{\mathcal{E}}$. It is exactly the Nakajima monomial set $\mathcal{M}_{c}$ introduced in [9] if we identify $Y_{i}(m)^{\left(0, y_{i}^{1}(m)\right)} \in \dot{\mathcal{M}}_{c}^{\mathcal{E}}$ with $Y_{i}(m)^{y_{i}^{1}(m)} \in \mathcal{M}_{c}$. The crystal structure on $\mathcal{M}_{c}$ is compatible with that on $\mathcal{M}_{c}^{\mathcal{E}}$ under this identification. We would like to mention that $\mathcal{M}_{c}$ can be treated as a subcrystal of $\mathcal{M}_{c}^{\mathcal{E}}$. Viewing $\mathcal{M}_{c}$ as a subcrystal of $\mathcal{M}_{c}^{\mathcal{E}}$, the monomial theory developed for irreducible highest weight crystal can easily be transferred to that on the extended monomial set.

We obtain the following statement from Theorem 2.1.

Corollary 2.3. ([13]) For a maximal vector $M \in \dot{\mathcal{M}}_{c}^{\mathcal{E}}$, the connected component of $\dot{\mathcal{M}}_{c}^{\mathcal{E}}$ containing $M$ is crystal isomorphic to $\mathcal{B}(\operatorname{wt}(M))$.

In the final section, we will give a concrete listing of elements belonging to the connected component containing a maximal vector $M \in \dot{\mathcal{M}}_{c}^{\mathcal{E}}$ mentioned in the above corollary, for the case of $A_{n}$. 


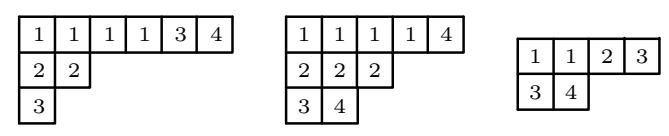

Figure 1: Large (left), marginally large (middle), and non-large (right) tableaux

Unless there is possibility of confusion, we shall omit $c$ and use the notations $\mathcal{M}, \dot{\mathcal{M}}^{\mathcal{E}}$, and $\mathcal{M}^{\mathcal{E}}$ instead of $\mathcal{M}_{c}, \dot{\mathcal{M}}_{c}^{\mathcal{E}}$, and $\mathcal{M}_{c}^{\mathcal{E}}$, respectively.

\subsection{Young tableaux}

In this section, we recall a Young tableau description of the crystals $\mathcal{B}(\infty)$ and $\mathcal{B}(\lambda)$ for type $A_{n}$, that are crucial for our work. Using these, in the remaining sections, we shall show that the sets of monomials, satisfying some appropriate conditions, give new descriptions of $\mathcal{B}(\infty)$ and $\mathcal{B}(\lambda)$.

Based on result of the paper [10], we shall identify elements of the highest weight crystal $\mathcal{B}(\lambda)$ with semistandard tableaux of $\lambda$-shape, for the $A_{n}$ case. Since this work is a well known result, we refer readers to the original papers and shall not repeat the complicated definitions here.

\section{DEFINITION 2.4.}

(1) A semistandard tableau $T$ of shape $\lambda \in P^{+}$, equivalently, an element of an irreducible highest weight crystal $\mathcal{B}(\lambda)$ for the $A_{n}$ type, is large if it consists of $n$ non-empty rows, and if for each $1 \leq i \leq n$, the number of $i$-boxes in the $i$-th row is strictly greater than the number of all boxes in the $(i+1)$-th row. In particular the $n$-th row of $T$ contains at least one $n$-box.

(2) A large tableau $T$ is marginally large if for $1 \leq i \leq n$, the number of $i$-boxes in the $i$-th row of $T$ is greater than the number of all boxes in the $(i+1)$-th row by exactly one. In particular, the $n$-th row of $T$ should contain one $n$-box.

In Figure 1, for $A_{3}$ type, we give examples of semistandard tableaux. The one on the left is large, the one in the middle is marginally large, and the one on the right is not large.

Definition 2.5. We denote by $\mathcal{T}(\infty)$ the set of all marginally large tableaux. The marginally large tableau whose $i$-th row consists only of $i$-boxes $(i \in I)$ is denoted by $T_{\infty}$. 
ExAmple 2.6. The set $\mathcal{T}(\infty)$ for case $A_{3}$, consists of all tableaux of the following form. The unshaded part must exist, whereas the shaded part is optional with variable size.

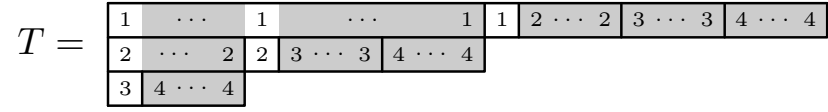

$$
\begin{aligned}
& T_{\infty}=\begin{array}{|l|l|l|}
\hline 1 & 1 & 1 \\
\hline 2 & 2 \\
\hline 3 & \multicolumn{1}{|l}{} \\
\hline
\end{array}
\end{aligned}
$$

We recall the action of Kashiwara operators $\tilde{f}_{i}, \tilde{e}_{i}(i \in I)$ on marginally large tableaux $T \in \mathcal{T}(\infty)$.

(1) We first read the boxes in the tableau $T$ through the far eastern reading and write down the boxes in tensor product form. That is, we read through each column from top to bottom starting from the rightmost column, continuing to the left, and lay down the read boxes from left to right in tensor product form. The following diagram gives an example.

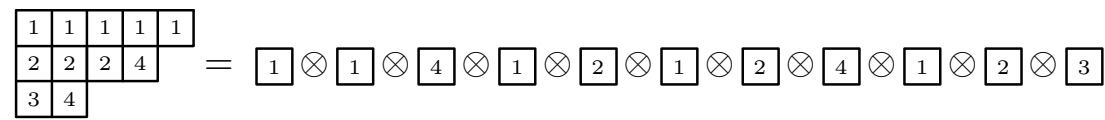

(2) Under each tensor component $x$ of $T$, write down $\varepsilon_{i}(x)$-many 1 s followed by $\varphi_{i}(x)$-many $0 \mathrm{~s}$. Then, from the long sequence of mixed $0 \mathrm{~s}$ and 1 s, successively cancel out every occurrence of $(0,1)$ pair until we arrive at a sequence of $1 \mathrm{~s}$ followed by $0 \mathrm{~s}$, reading from left to right. This is called the $i$-signature of $T$.

(3) Denote by $T^{\prime}$, the tableau obtained from $T$, by replacing the box $x$ corresponding to the leftmost 0 in the $i$-signature of $T$ with the box $\tilde{f}_{i} x$.

- If $T^{\prime}$ is a large tableau, it is automatically marginally large. We define $\tilde{f}_{i} T$ to be $T^{\prime}$.

- If $T^{\prime}$ is not large, then we define $\tilde{f}_{i} T$ to be the large tableau obtained by inserting one column consisting of $i$ rows to the left of the box $\tilde{f}_{i}$ acted upon. The added column should have a $k$-box at the $k$-th row for $1 \leq k \leq i$.

(4) Denote by $T^{\prime}$, the tableau obtained from $T$, by replacing the box $x$ corresponding to the rightmost 1 in the $i$-signature of $T$ with the box $\tilde{e}_{i} x$. 
- If $T^{\prime}$ is a marginally large tableau, then we define $\tilde{e}_{i} T$ to be $T^{\prime}$.

- If $T^{\prime}$ is large but not marginally large, then we define $\tilde{e}_{i} T$ to be the large tableau obtained by removing the column containing the changed box. It will be of $i$ rows and have a $k$-box at the $k$-th row for $1 \leq k \leq i$.

(5) If there is no 1 in the $i$-signature of $T$, we define $\tilde{e}_{i} T=0$.

Remark 2.7. The condition large imposed on the tableau $T$ ensures that its $i$-signature always contains 0 's.

Let $T$ be a tableau in $\mathcal{T}(\infty)$ with the $i$-th row, for each $1 \leq i \leq n$, consisting of $b_{j}^{i}$-many $j \mathrm{~s}(i<j \leq n+1)$ and some number of $i$ s. We define the maps wt $: \mathcal{T}(\infty) \rightarrow P, \varphi_{i}, \varepsilon_{i}: \mathcal{T}(\infty) \rightarrow \mathbf{Z}$ by setting

$$
\begin{aligned}
\operatorname{wt}(T) & =-\sum_{j=1}^{n}\left(\sum_{k=j+1}^{n+1} b_{k}^{1}+\sum_{k=j+1}^{n+1} b_{k}^{2}+\cdots+\sum_{k=j+1}^{n+1} b_{k}^{j}\right) \alpha_{j}, \\
\varepsilon_{i}(T) & =\text { the number of } 1 \text { s in the } i \text {-signature of } T, \\
\varphi_{i}(T) & =\varepsilon_{i}(T)+\left\langle h_{i}, \operatorname{wt}(T)\right\rangle .
\end{aligned}
$$

TheOREm 2.8. ([3]) The Kashiwara operators and various maps given above define a crystal structure on $\mathcal{T}(\infty)$. The crystal $\mathcal{T}(\infty)$ is isomorphic to $\mathcal{B}(\infty)$ as $U_{q}\left(A_{n}\right)$-crystal.

In Figure 2, we illustrate the top part of the crystal $\mathcal{T}(\infty)$ for type $A_{2}$.

\section{§3. Monomial description of crystal $\mathcal{B}(\infty)$}

We give an explicit description of the crystal $\mathcal{B}(\infty)$ for $A_{n}$-type, in terms of extended Nakajima monomials. We first present a candidate monomial set, show this set to be a crystal, and give a crystal isomorphism of this with another description of $\mathcal{B}(\infty)$.

We take the set $c=\left(c_{i j}\right)_{i \neq j}$ to be

$$
c_{i j}= \begin{cases}0 & \text { if } i>j, \\ 1 & \text { if } i<j .\end{cases}
$$

Then for $i \in I$ and $m \in \mathbf{Z}$, we have

$$
A_{i}(m)^{ \pm 1}=Y_{i}(m)^{(0, \pm 1)} Y_{i}(m+1)^{(0, \pm 1)} Y_{i-1}(m+1)^{(0, \mp 1)} Y_{i+1}(m)^{(0, \mp 1)} .
$$




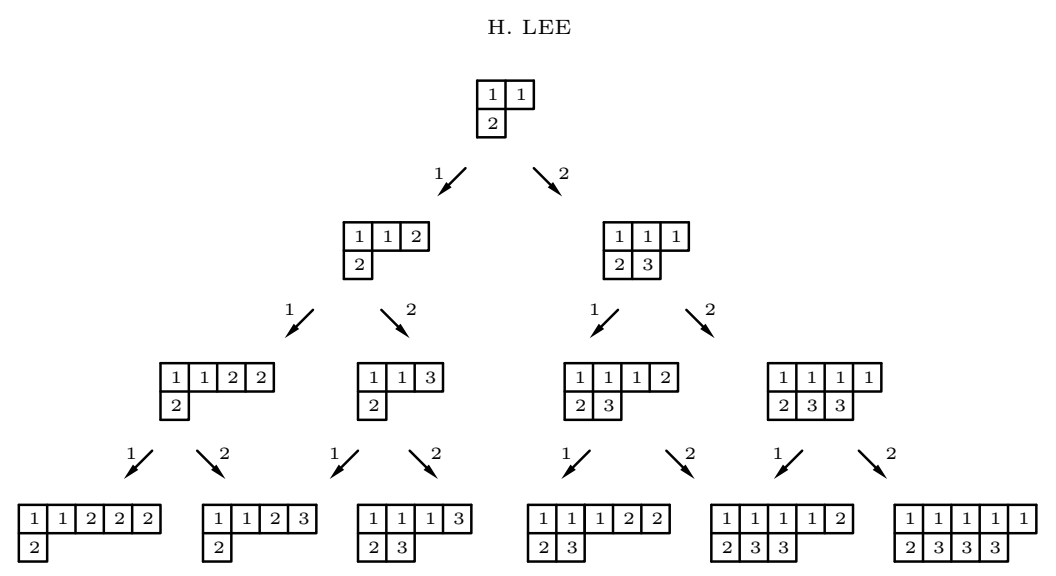

Figure 2: Part of the crystal $\mathcal{T}(\infty)$ for type $A_{2}$

Here, we are setting $Y_{0}(k)^{(0, \pm 1)}=Y_{n+1}(k)^{(0, \pm 1)}=1$.

As was stated in Remark 2.2, the theory to be developed on the fixed $\mathcal{M}_{c}^{\mathcal{E}}$ can similarly be developed on all the other crystals induced from the extended Nakajima monomial set through other choices of the set $c$, under the isomorphism given in Proposition 3.2 (2) of [13]. From now on, we shall omit $c$ and use the notation $\mathcal{M}^{\mathcal{E}}$ instead of $\mathcal{M}_{c}^{\mathcal{E}}$, since we already fixed the set $c$.

The set we define below is originally obtained by applying Kashiwara operators $\tilde{f}_{i}(i \in I)$ iteratively, starting from the maximal element $\prod_{i \in I} Y_{i}(-i)^{(1,0)} \in \mathcal{M}^{\mathcal{E}}$ of $\tilde{\mathrm{wt}}\left(\prod_{i \in I} Y_{i}(-i)^{(1,0)}\right)=\sum_{i}(1,0) \Lambda_{i}$. This choice of starting monomial will allow us to relate monomials of the set defined below to tableaux in $\mathcal{T}(\infty)$ naturally.

Definition 3.1. Consider elements of $\mathcal{M}^{\mathcal{E}}$ having the form

$$
\begin{aligned}
M= & \prod_{i \in I}\left(Y_{i}(-i)^{\left(1, a_{i}^{i}\right)} \prod_{m=0}^{i-1} Y_{i}(-m)^{\left(0, a_{i}^{m}\right)}\right) \\
= & Y_{1}(-1)^{\left(1, a_{1}^{1}\right)} Y_{1}(0)^{\left(0, a_{1}^{0}\right)} \\
& \cdot Y_{2}(-2)^{\left(1, a_{2}^{2}\right)} Y_{2}(-1)^{\left(0, a_{2}^{1}\right)} Y_{2}(0)^{\left(0, a_{2}^{0}\right)} \\
& \cdots \\
& \cdot Y_{n}(-n)^{\left(1, a_{n}^{n}\right)} Y_{n}(-n+1)^{\left(0, a_{n}^{n-1}\right)} \cdots Y_{n}(-1)^{\left(0, a_{n}^{1}\right)} Y_{n}(0)^{\left(0, a_{n}^{0}\right)}
\end{aligned}
$$

with the conditions 


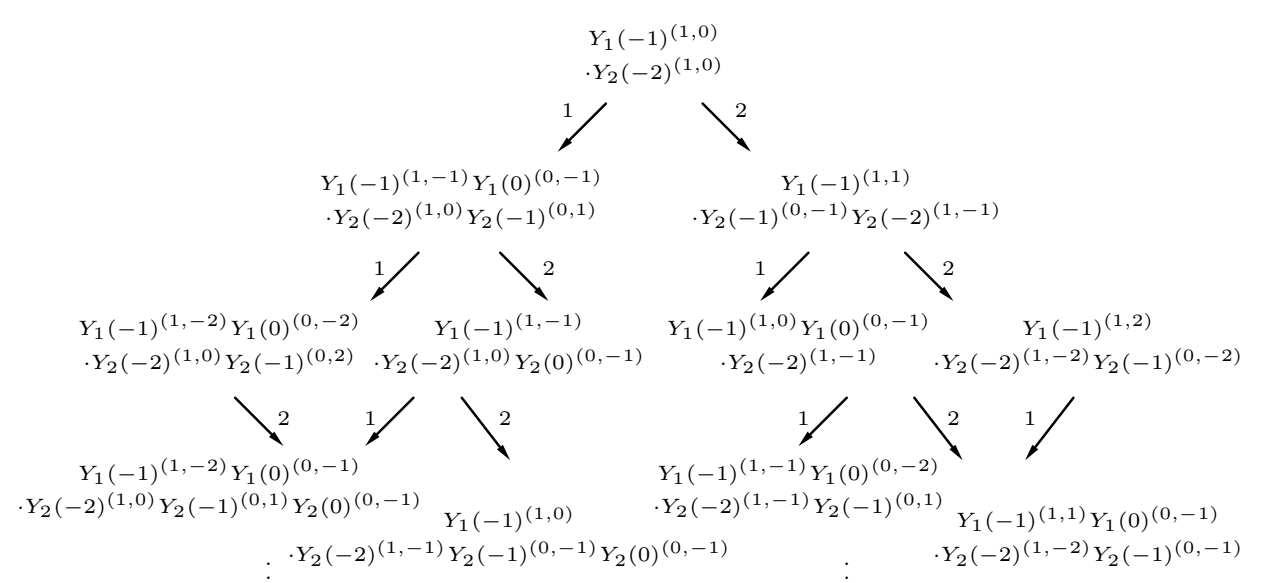

Figure 3: Part of the monomial set $\mathcal{M}(\infty)$ for $A_{2}$ type

(1) $\sum_{j=0}^{k} a_{i+j}^{j} \leq 0$ for each $0 \leq k \leq n-1,1 \leq i \leq n-k$,

(2) $\sum_{i=1}^{n-k}\left(\sum_{j=0}^{k} a_{i+j}^{j}\right)=\sum_{i=k+1}^{n} a_{i}^{i}$ for $0 \leq k \leq n-1$.

Specifically, in case of $a_{i}^{j}=0$ for all $i, j$, we have

$$
M=\prod_{i \in I} Y_{i}(-i)^{(1,0)}=Y_{1}(-1)^{(1,0)} Y_{2}(-2)^{(1,0)} \cdots Y_{n}(-n)^{(1,0)} .
$$

We denote by $\mathcal{M}(\infty)$ the set of all monomials of the form (3.2) and by $M_{\infty}$ the monomial of (3.3).

Actually, as we will become apparent later, this set $\mathcal{M}(\infty)$ is closed and connected under Kashiwara operators (2.11) and (2.12) on $\mathcal{M}^{\mathcal{E}}$. Figure 3 is the top part of monomial set $\mathcal{M}(\infty)$.

We now introduce new expressions for elements of $\mathcal{M}(\infty)$. First, we introduce the following notation.

Definition 3.2. For $u, v, m \in \mathbf{Z}$, and $1 \leq i \leq n+1$, we use the notation

$$
X_{i}(m)^{(u, v)}=Y_{i}(m)^{(u, v)} Y_{i-1}(m+1)^{(-u,-v)} .
$$

Here, we set $Y_{0}(k)^{(u, v)}=Y_{n+1}(k)^{(u, v)}=1$. 
Remark 3.3. From the above notation, we obtain

$$
\begin{aligned}
Y_{i}(m)^{(u, v)}= & X_{1}(m+i-1)^{(u, v)} X_{2}(m+i-2)^{(u, v)} \cdots X_{i}(m)^{(u, v)} \\
= & X_{n+1}(m+i-(n+1))^{(-u,-v)} X_{n}(m+i-n)^{(-u,-v)} \\
& \cdots X_{i+1}(m-1)^{(-u,-v)}
\end{aligned}
$$

for each $i \in I$. And so, we may write

$$
A_{i}(m)=X_{i}(m)^{(0,1)} X_{i+1}(m)^{(0,-1)} .
$$

This is very useful when computing Kashiwara action on monomials written in terms of $X_{i}(m)^{(u, v)}$.

Proposition 3.4. Consider elements of $\mathcal{M}^{\mathcal{E}}$ having the form

$$
\begin{aligned}
M= & \prod_{i \in I}\left(X_{i}(-i)^{\left(n-i+1,-\sum_{k=i+1}^{n+1} b_{k}^{i}\right)} \prod_{k=i+1}^{n+1} X_{k}(-i)^{\left(0, b_{k}^{i}\right)}\right) \\
= & X_{1}(-1)^{\left(n,-\sum_{k=2}^{n+1} b_{k}^{1}\right)} X_{2}(-1)^{\left(0, b_{2}^{1}\right)} \cdots X_{n}(-1)^{\left(0, b_{n}^{1}\right)} X_{n+1}(-1)^{\left(0, b_{n+1}^{1}\right)} \\
& \cdot X_{2}(-2)^{\left(n-1,-\sum_{k=3}^{n+1} b_{k}^{2}\right)} X_{3}(-2)^{\left(0, b_{3}^{2}\right)} \cdots X_{n+1}(-2)^{\left(0, b_{n+1}^{2}\right)} \\
& \cdots \\
& \cdot X_{n-1}(-n+1)^{\left(2,-\sum_{k=n}^{n+1} b_{k}^{n-1}\right)} X_{n}(-n+1)^{\left(0, b_{n}^{n-1}\right)} X_{n+1}(-n+1)^{\left(0, b_{n+1}^{n-1}\right)} \\
& \cdot X_{n}(-n)^{\left(1,-b_{n+1}^{n}\right)} X_{n+1}(-n)^{\left(0, b_{n+1}^{n}\right)}
\end{aligned}
$$

where $b_{k}^{i} \geq 0$ for all $k, i$. Each element of $\mathcal{M}(\infty)$ may be written uniquely in this form. Conversely, any element of this form is an element of $\mathcal{M}(\infty)$.

Proof. Given any monomial

$$
M=\prod_{i \in I}\left(Y_{i}(-i)^{\left(1, a_{i}^{i}\right)} \prod_{m=0}^{i-1} Y_{i}(-m)^{\left(0, a_{i}^{m}\right)}\right) \in \mathcal{M}(\infty),
$$

through routine computation using (3.4), we can obtain the expression

$$
\begin{aligned}
& M=X_{1}(-1)^{\left(n, \sum_{i=1}^{n} a_{i}^{i}\right)} X_{2}(-1)^{\left(0,-a_{1}^{0}\right)} X_{3}(-1)^{\left(0,-a_{2}^{0}\right)} \cdots X_{n+1}(-1)^{\left(0,-a_{n}^{0}\right)} \\
& \cdot X_{2}(-2)^{\left(n-1, \sum_{i=2}^{n} a_{i}^{i}\right)} X_{3}(-2)^{\left(0,-a_{1}^{0}-a_{2}^{1}\right)} X_{4}(-2)^{\left(0,-a_{2}^{0}-a_{3}^{1}\right)} \cdots X_{n+1}(-2)^{\left(0,-a_{n-1}^{0}-a_{n}^{1}\right)} \\
& \cdots \\
& \cdot X_{n-1}(-n+1)^{\left(2, \sum_{i=n-1}^{n} a_{i}^{i}\right)} X_{n}(-n+1)^{\left(0,-\sum_{i=0}^{n-2} a_{i+1}^{i}\right)} X_{n+1}(-n+1)^{\left(0,-\sum_{i=0}^{n-2} a_{i+2}^{i}\right)} \\
& \cdot X_{n}(-n)^{\left(1, a_{n}^{n}\right)} X_{n+1}(-n)^{\left(0,-\sum_{i=0}^{n-1} a_{i+1}^{i}\right)} .
\end{aligned}
$$


Since $M \in \mathcal{M}(\infty)$, from the conditions given in (3.2), we obtain the form given in (3.5). The element $M_{\infty}=\prod_{i \in I} Y_{i}(-i)^{(1,0)}$ can be rewritten in the form $\prod_{i \in I} X_{i}(-i)^{(n-i+1,0)}$.

Conversely, given any monomial of the form (3.5), we have

$$
\begin{aligned}
M= & Y_{1}(-1)^{\left(1,-\sum_{k=2}^{n+1} b_{k}^{1}+\sum_{k=3}^{n+1} b_{k}^{2}\right)} Y_{1}(0)^{\left(0,-b_{2}^{1}\right)} \\
& \cdot Y_{2}(-2)^{\left(1,-\sum_{k=3}^{n+1} b_{k}^{2}+\sum_{k=4}^{n+1} b_{k}^{3}\right)} Y_{2}(-1)^{\left(0, b_{2}^{1}-b_{3}^{2}\right)} Y_{2}(0)^{\left(0,-b_{3}^{1}\right)} \\
& \cdots \\
& \cdot Y_{n-1}(-n+1)^{\left(1,-\sum_{k=n}^{n+1} b_{k}^{n-1}+\sum_{k=n+1}^{n+1} b_{k}^{n}\right)} Y_{n-1}(-n+2)^{\left(0, b_{n-1}^{n-2}-b_{n}^{n-1}\right)} \\
& \quad \cdots Y_{n-1}(-1)^{\left(0, b_{n-1}^{1}-b_{n}^{2}\right)} Y_{n-1}(0)^{\left(0,-b_{n}^{1}\right)} \\
& \cdot Y_{n}(-n)^{\left(1,-b_{n+1}^{n}\right)} Y_{n}(-n+1)^{\left(0, b_{n}^{n-1}-b_{n+1}^{n}\right)} \\
& \quad \cdots Y_{n}(-2)^{\left(0, b_{n}^{2}-b_{n+1}^{3}\right)} Y_{n}(-1)^{\left(0, b_{n}^{1}-b_{n+1}^{2}\right)} Y_{n}(0)^{\left(0,-b_{n+1}^{1}\right)} .
\end{aligned}
$$

It is now straightforward to check that $M \in \mathcal{M}(\infty)$. We have thus shown that $\mathcal{M}(\infty)$ consists of elements of the form (3.5).

The uniqueness part may be proved through simple computation.

Remark 3.5. There are other ways to write each element of $\mathcal{M}(\infty)$ as products of the terms $X_{j}(m)^{(u, v)}$. The product form (3.5) was chosen because it allows us to relate monomials of the set $\mathcal{M}(\infty)$ to tableaux in $\mathcal{T}(\infty)$ directly.

Now, we translate the Kashiwara operator actions (2.11), (2.12) into a form suitable for the new monomial expression of $\mathcal{M}(\infty)$.

LEMma 3.6. The set $\mathcal{M}(\infty)$ is closed under the action given below: Fix element

$$
M=\prod_{i \in I}\left(X_{i}(-i)^{\left(n-i+1,-\sum_{k=i+1}^{n+1} b_{k}^{i}\right)} \prod_{k=i+1}^{n+1} X_{k}(-i)^{\left(0, b_{k}^{i}\right)}\right) \in \mathcal{M}(\infty) .
$$

Consider the following finite ordered sequence of some components of $M$ :

$$
\begin{gathered}
X_{n+1}(-1)^{\left(0, b_{n+1}^{1}\right)}, X_{n}(-1)^{\left(0, b_{n}^{1}\right)}, \ldots, X_{2}(-1)^{\left(0, b_{2}^{1}\right)}, \\
X_{n+1}(-2)^{\left(0, b_{n+1}^{2}\right)}, X_{n}(-2)^{\left(0, b_{n}^{2}\right)}, \ldots, X_{3}(-2)^{\left(0, b_{3}^{2}\right)}, \\
\ldots, \\
X_{n+1}(-n+1)^{\left(0, b_{n+1}^{n-1}\right)}, X_{n}(-n+1)^{\left(0, b_{n}^{n-1}\right)}, \\
X_{n+1}(-n)^{\left(0, b_{n+1}^{n}\right)} .
\end{gathered}
$$


(1) For $i \in I$, under each component $X_{i+1}(-m)^{\left(0, b_{i+1}^{m}\right)}$ of the above sequence, write $b_{i+1}^{m}$-many 1 's, and under each $X_{i}(-m)^{\left(0, b_{i}^{m}\right)}$, write $b_{i}^{m}$ many 0's.

(2) From this sequence of 1's and 0 's, successively cancel out each $(0,1)$ pair to obtain a sequence of 1 's followed by 0 's (reading from left to right). This remaining 1 's and 0 's sequence is called the $i$-signature of $M$.

(3) We define

$$
\tilde{f}_{i} M=M \cdot X_{i}(-m)^{(0,-1)} X_{i+1}(-m)^{(0,1)}=M A_{i}(-m)^{-1}
$$

if the component $X_{i}(-m)^{\left(0, b_{i}^{m}\right)}$ corresponds to the left-most 0 of the $i$-signature of $M$ and

$$
\tilde{e}_{i} M=M \cdot X_{i}(-m)^{(0,1)} X_{i+1}(-m)^{(0,-1)}=M A_{i}(-m)
$$

if the component $X_{i+1}(-m)^{\left(0, b_{i+1}^{m}\right)}$ corresponds to the right-most 1 .

(4) We define $\tilde{e}_{i} M=0$ if no 1 remains and

$$
\tilde{f}_{i} M=M \cdot X_{i}(-i)^{(0,-1)} X_{i+1}(-i)^{(0,1)}=M A_{i}(-i)^{-1}
$$

if no 0 remains.

Proof. We show that the actions satisfy the following properties:

$$
\tilde{f}_{i} \mathcal{M}(\infty) \subset \mathcal{M}(\infty), \quad \tilde{e}_{i} \mathcal{M}(\infty) \subset \mathcal{M}(\infty) \cup\{0\} \quad \text { for all } i \in I .
$$

For $M \in \mathcal{M}(\infty)$, if the $i$-signature of $M$ contains at least one 0 , then the left-most 0 of the $i$-signature of $M$ corresponds to a component $X_{i}(-m)^{\left(0, b_{i}^{m}\right)}$ for some $m=1, \ldots, i-1$ and the exponent of component $X_{i}(-m)^{\left(0, b_{i}^{m}\right)}$ corresponding to the left-most 0 has the property $\left(0, b_{i}^{m}\right) \geq(0,1)$. Thus the monomial $\tilde{f}_{i} M=M X_{i}(-m)^{(0,-1)} X_{i+1}(-m)^{(0,1)}$ defined in (3.8) is contained in $\mathcal{M}(\infty)$.

When the $i$-signature of $M$ contains no 0 , we define $\tilde{f}_{i} M$ as in (3.9). Since the exponents of the components $X_{i}(-i)$ of $M$ has the property $\geq$ $(0,1), \tilde{f}_{i} M$ given in (3.9) also are in $\mathcal{M}(\infty)$. So the set $\mathcal{M}(\infty)$ is closed under the above operator $\tilde{f}_{i}$.

Proof for the statements concerning $\tilde{e}_{i}$ may be done in a similar manner. 
LEMmA 3.7. The operation given in Lemma 3.6 is just another expression of the Kashiwara operators given on $\mathcal{M}^{\mathcal{E}}$, restricted to $\mathcal{M}(\infty)$.

Proof. As we can see in equations (3.8) to (3.9), for each $M \in \mathcal{M}(\infty)$, $\tilde{f}_{i} M$ can also be expressed in form $M A_{i}(-m)^{-1}(m=1,2, \ldots, i)$. To show that this operation is just another interpretation of the Kashiwara operator $\tilde{f}_{i}$ given on $\mathcal{M}^{\mathcal{E}}$, restricted to $\mathcal{M}(\infty)$, it is enough to show that $m_{f}$ defined in (2.13) for each $M$ is equal to $-m$ of $M A_{i}(-m)^{-1}$ given in equations (3.8) to (3.9). Note that we can easily see that from $M$ given by expression (3.2), for each $M \in \mathcal{M}(\infty), \tilde{\varphi}_{i}(M)>(0,0)$.

Given a monomial $M \in \mathcal{M}(\infty)$, we can express it in the following two forms.

$$
\begin{aligned}
M= & \prod_{i \in I}\left(X_{i}(-i)^{\left(n-i+1,-\sum_{k=i+1}^{n+1} b_{k}^{i}\right)} \prod_{k=i+1}^{n+1} X_{k}(-i)^{\left(0, b_{k}^{i}\right)}\right) \\
= & Y_{1}(-1)^{\left(1,-\sum_{k=2}^{n+1} b_{k}^{1}+\sum_{k=3}^{n+1} b_{k}^{2}\right)} Y_{1}(0)^{\left(0,-b_{2}^{1}\right)} \\
& \cdots \\
& \cdot Y_{n-1}(-n+1)^{\left(1,-\sum_{k=n}^{n+1} b_{k}^{n-1}+\sum_{k=n+1}^{n+1} b_{k}^{n}\right)} Y_{n-1}(-n+2)^{\left(0, b_{n-1}^{n-2}-b_{n}^{n-1}\right)} \\
& \quad \cdots Y_{n-1}(-1)^{\left(0, b_{n-1}^{1}-b_{n}^{2}\right)} Y_{n-1}(0)^{\left(0,-b_{n}^{1}\right)} \\
& \cdot Y_{n}(-n)^{\left(1,-b_{n+1}^{n}\right)} Y_{n}(-n+1)^{\left(0, b_{n}^{n-1}-b_{n+1}^{n}\right)} \\
& \cdots Y_{n}(-1)^{\left(0, b_{n}^{1}-b_{n+1}^{2}\right)} Y_{n}(0)^{\left(0,-b_{n+1}^{1}\right)}
\end{aligned}
$$

If the $i$-signature of $M$ contains at least one 0 and $X_{i}(-m)^{\left(0, b_{i}^{m}\right)}$ is the component corresponding to the left-most 0 in the $i$-signature of $M$, then $-m$ is one of $-1,-2, \ldots,-i+1$ and we can obtain

$$
-m=\min \left\{j \mid \max \left\{\sum_{k \leq j} y_{i}(k) \mid j \in \mathbf{Z}\right\}\right\}=m_{f}
$$

where $y_{i}(k)$ is the exponent of $Y_{i}(k)$ appearing in $M$ given by expression (3.11).

If the $i$-signature of $M$ contains no 0 ,

$$
-i=\min \left\{j \mid \max \left\{\sum_{k \leq j} y_{i}(k) \mid j \in \mathbf{Z}\right\}\right\}=m_{f} .
$$

In all cases, we can confirm that $m_{f}=-m$, where $-m$ is given through equations (3.8) to (3.9) stating $\tilde{f}_{i} M=M A_{i}(-m)^{-1}$. Proof for the statements concerning $\tilde{e}_{i}$ may be done in a similar manner. 


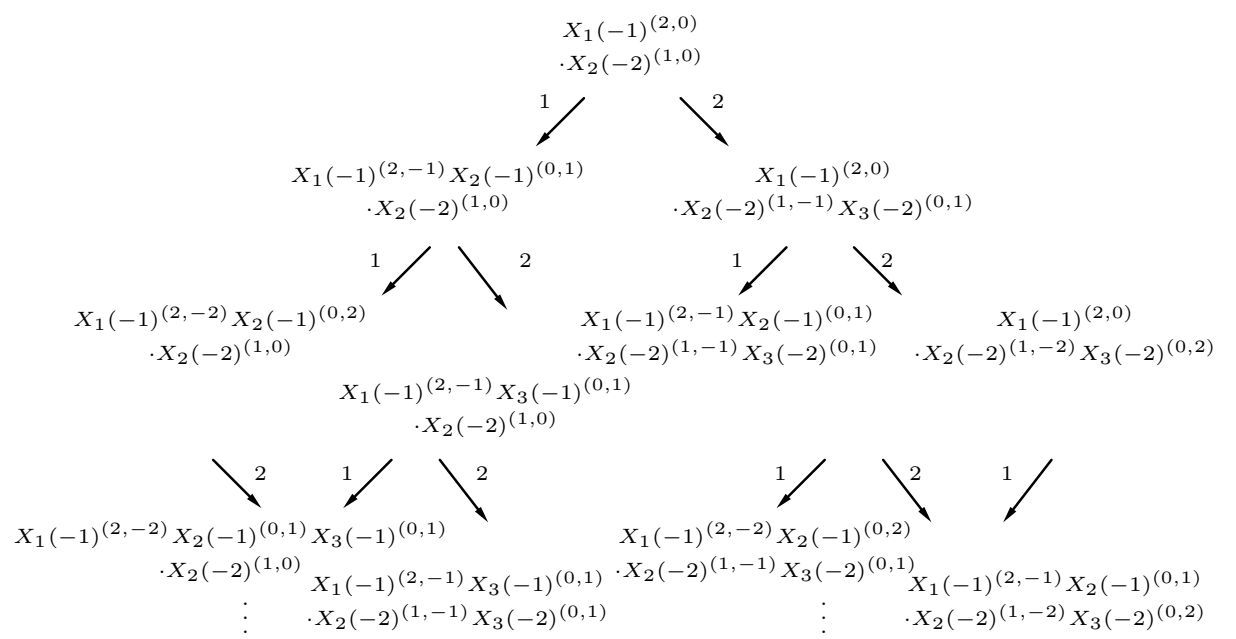

Figure 4: Part of crystal $\mathcal{M}(\infty)$ for type $A_{2}$

From the above lemmas, we obtain the following result.

Proposition 3.8. The set $\mathcal{M}(\infty)$ forms a $U_{q}\left(A_{n}\right)$-subcrystal of $\mathcal{M}^{\mathcal{E}}$.

Figures 4 illustrates the top part of crystal $\mathcal{M}(\infty)$ for type $A_{2}$. It was obtained by applying the Kashiwara actions introduced in Lemma 3.6 on the new expression for elements of $\mathcal{M}(\infty)$. Readers may want to compare this with Figures 2 and 3.

The crystal structure of $\mathcal{M}^{\mathcal{E}}$ allows us to obtain more general results that will be introduced below.

Definition 3.9. Fix any set of positive integers $p_{i}$ and any integer $r$. Consider elements of $\mathcal{M}^{\mathcal{E}}$ having the form

$$
\begin{aligned}
M= & \prod_{i \in I}\left(Y_{i}(r-i)^{\left(p_{i}, a_{i}^{i}\right)} \prod_{m=0}^{i-1} Y_{i}(r-m)^{\left(0, a_{i}^{m}\right)}\right) \\
= & Y_{1}(r-1)^{\left(p_{1}, a_{1}^{1}\right)} Y_{1}(r)^{\left(0, a_{1}^{0}\right)} \\
& \cdot Y_{2}(r-2)^{\left(p_{2}, a_{2}^{2}\right)} Y_{2}(r-1)^{\left(0, a_{2}^{1}\right)} Y_{2}(r)^{\left(0, a_{2}^{0}\right)} \\
& \cdots \\
& \cdot Y_{n}(r-n)^{\left(p_{n}, a_{n}^{n}\right)} Y_{n}(r-n+1)^{\left(0, a_{n}^{n-1}\right)} \cdots Y_{n}(r-1)^{\left(0, a_{n}^{1}\right)} Y_{n}(r)^{\left(0, a_{n}^{0}\right)}
\end{aligned}
$$


and satisfying the same condition given to (3.2). In case of $a_{i}^{j}=0$ for all $i$, $j$, we have

$$
M=\prod_{i \in I} Y_{i}(r-i)^{\left(p_{i}, 0\right)} .
$$

We denote by $\mathcal{M}\left(p_{1}, \ldots, p_{n} ; r ; \infty\right)$ the set of all monomials of the form (3.13) and by $M_{\left(p_{1}, \ldots, p_{n} ; r ; \infty\right)}$ the monomial of $(3.14)$.

A result similar to Proposition 3.5 may be obtained for $\mathcal{M}\left(p_{1}, \ldots, p_{n}\right.$; $r ; \infty)$.

Proposition 3.10. Each element of $\mathcal{M}\left(p_{1}, \ldots, p_{n} ; r ; \infty\right)$ may be written uniquely in this form

$$
M=\prod_{i \in I}\left(X_{i}(r-i)^{\left(\sum_{k=i}^{n} p_{k},-\sum_{k=i+1}^{n+1} b_{k}^{i}\right)} \prod_{k=i+1}^{n+1} X_{k}(r-i)^{\left(0, b_{k}^{i}\right)}\right)
$$

where $b_{k}^{i} \geq 0$ for all $k, i$. Conversely, any element in $\mathcal{M}^{\mathcal{E}}$ of this form is an element of $\mathcal{M}\left(p_{1}, \ldots, p_{n} ; r ; \infty\right)$.

We believe the readers can easily write down the process for change of variable similar to that given by (3.6) and (3.7) for $\mathcal{M}\left(p_{1}, \ldots, p_{n} ; r ; \infty\right)$.

The set $\mathcal{M}(\infty)$ is a special case of this set $\mathcal{M}\left(p_{1}, \ldots, p_{n} ; r ; \infty\right)$ which is $r=0$ and $p_{i}=1$ for all $i \in I$.

Remark 3.11. It is possible to obtain results similar to Lemmas 3.6 and 3.7 also for the case $\mathcal{M}\left(p_{1}, \ldots, p_{n} ; r ; \infty\right)$ in a manner similar to that for $\mathcal{M}(\infty)$. Thus we can state that the set $\mathcal{M}\left(p_{1}, \ldots, p_{n} ; r ; \infty\right)$ forms a subcrystal of $\mathcal{M}^{\mathcal{E}}$.

Proposition 3.12. The set $\mathcal{M}\left(p_{1}, \ldots, p_{n} ; r ; \infty\right)$ forms a subcrystal of $\mathcal{M}^{\mathcal{E}}$ isomorphic to $\mathcal{M}(\infty)$ as $U_{q}\left(A_{n}\right)$-crystal.

Proof. As mentioned in Remark 3.11, the set $\mathcal{M}\left(p_{1}, \ldots, p_{n} ; r ; \infty\right)$ forms a subcrystal of $\mathcal{M}^{\mathcal{E}}$. Now let us show that the crystal $\mathcal{M}\left(p_{1}, \ldots, p_{n} ; r ; \infty\right)$ is isomorphic to $\mathcal{M}(\infty)$.

First, we define a canonical map $\phi: \mathcal{M}(\infty) \rightarrow \mathcal{M}\left(p_{1}, \ldots, p_{n} ; r ; \infty\right)$ by setting

$$
\phi(M)=\prod_{i \in I}\left(X_{i}(r-i)^{\left(\sum_{k=i}^{n} p_{k},-\sum_{k=i+1}^{n+1} b_{k}^{i}\right)} \prod_{k=i+1}^{n+1} X_{k}(r-i)^{\left(0, b_{k}^{i}\right)}\right)
$$


for $M$ of the form (3.5). The monomial $M_{\infty}$ of $\mathcal{M}(\infty)$ is mapped onto the vector $M_{\left(p_{1}, \ldots, p_{n} ; r ; \infty\right)}$. It is obvious that this map $\phi$ is well-defined and that it is actually bijective.

Note that the monomial of $(3.13)$ is the element of $\mathcal{M}\left(p_{1}, \ldots, p_{n} ; r ; \infty\right)$ corresponding to the monomial $(3.2)$ of $\mathcal{M}(\infty)$ under the map $\phi$.

The outputs of the functions wt, $\varphi_{i}$, and $\varepsilon_{i}$, defined in (2.8), (2.9), and (2.10) do not depend on $r$ or on any fixed positive integers $p_{i}$. Using Lemma 3.6 and its counterpart for $\mathcal{M}\left(p_{1}, \ldots, p_{n} ; r ; \infty\right)$, we can easily show that the map $\phi$ commutes with the Kashiwara operators. Hence, the set $\mathcal{M}\left(p_{1}, \ldots, p_{n} ; r ; \infty\right)$ forms a subcrystal of $\mathcal{M}^{\mathcal{E}}$ isomorphic to $\mathcal{M}(\infty)$.

Remark 3.13. It should be clear from the proof of Proposition 3.12, that in developing any theory for $\mathcal{M}\left(p_{1}, \ldots, p_{n} ; r ; \infty\right)$ the actual values of integer $r$ or $\left(p_{1}, \ldots, p_{n}\right)$ will not be very important. Arguments made for any set of such values can easily be adapted to applied to other set of such values. Hence, we shall concentrate on the theory for $\mathcal{M}(\infty)$ only.

Now, we will show that $\mathcal{M}(\infty)$ is a new description of $\mathcal{B}(\infty)$ by giving a crystal isomorphism. Recall that the crystal $\mathcal{T}(\infty)$ gives a realization of the crystal $\mathcal{B}(\infty)$ (see Theorem 2.8).

Here is one of our main theorem.

THEOREM 3.14. There exists a $U_{q}\left(A_{n}\right)$-crystal isomorphism

$$
\mathcal{T}(\infty) \stackrel{\sim}{\longrightarrow} \mathcal{M}(\infty)
$$

which maps $T_{\infty}$ to $M_{\infty}$. It means that the crystal $\mathcal{M}(\infty)$ is the connected component of $\mathcal{M}^{\mathcal{E}}$ containing the maximal vector $M_{\infty}$ of $\tilde{\mathrm{wt}}\left(M_{\infty}\right)=$ $\sum_{i}(1,0) \Lambda_{i}$ and is isomorphic to $\mathcal{B}(\infty)$.

Proof. We define a canonical map $\Phi: \mathcal{T}(\infty) \rightarrow \mathcal{M}(\infty)$ by setting, for each tableau $T \in \mathcal{T}(\infty)$ with $i$ th $(i \in I)$ row consists of $b_{j}^{i}$-many $j$-boxes, for each $i<j \leq n+1$, and some number of $i$-boxes,

$$
\Phi(T)=\prod_{i \in I}\left(X_{i}(-i)^{\left(n-i+1,-\sum_{k=i+1}^{n+1} b_{k}^{i}\right)} \prod_{k=i+1}^{n+1} X_{k}(-i)^{\left(0, b_{k}^{i}\right)}\right) \in \mathcal{M}(\infty) .
$$

It is obvious that this map $\Phi$ is well-defined and that it is actually bijective.

The action of Kashiwara operators on $\mathcal{M}(\infty)$ given in Lemma 3.6 follows the process for defining it on $\mathcal{T}(\infty)$. Hence, the map $\Phi$ naturally commutes with the Kashiwara operators $\tilde{f}_{i}$ and $\tilde{e}_{i}$. 
Remark 3.15. From Proposition 3.12 and Theorem 3.14, we can conclude that the crystal $\mathcal{M}\left(p_{1}, \ldots, p_{n} ; r ; \infty\right)$ is also isomorphic to $\mathcal{B}(\infty)$. It means that for positive integers $p_{i}$ and integer $r$, the crystal $\mathcal{M}\left(p_{1}, \ldots, p_{n}\right.$; $r ; \infty)$ is the connected component of $\mathcal{M}^{\mathcal{E}}$ containing the maximal vector $M_{\left(p_{1}, \ldots, p_{n} ; r ; \infty\right)} \in \mathcal{M}^{\mathcal{E}}$ of $\tilde{\operatorname{wt}}\left(M_{\left(p_{1}, \ldots, p_{n} ; r ; \infty\right)}\right)=\sum_{i \in I}\left(p_{i}, 0\right) \Lambda_{i}$ and is isomorphic to $\mathcal{B}(\infty)$.

EXAMPLE 3.16. We illustrate the correspondence between $\mathcal{T}(\infty)$ and $\mathcal{M}(\infty)$ for type $A_{3}$. Consider a monomial of $\mathcal{M}(\infty)$

$$
\begin{aligned}
M= & Y_{1}(-1)^{(1,-5)} Y_{1}(0)^{(0,-3)} \\
& \cdot Y_{2}(-2)^{(1,-1)} Y_{2}(-1)^{(0,1)} Y_{2}(0)^{(0,0)} \\
& \cdot Y_{3}(-3)^{(1,-1)} Y_{3}(-2)^{(0,1)} Y_{3}(-1)^{(0,0)} Y_{3}(0)^{(0,-4)} .
\end{aligned}
$$

It can be expressed as

$$
\begin{aligned}
M= & X_{4}(-4)^{(-1,5)} X_{3}(-3)^{(-1,5)} X_{2}(-2)^{(-1,5)} X_{4}(-3)^{(0,3)} X_{3}(-2)^{(0,3)} X_{2}(-1)^{(0,3)} \\
& \cdot X_{4}(-4)^{(-1,1)} X_{3}(-3)^{(-1,1)} X_{4}(-3)^{(0,-1)} X_{3}(-2)^{(0,-1)} \\
& \cdot X_{4}(-4)^{(-1,1)} X_{4}(-3)^{(0,-1)} X_{4}(-1)^{(0,4)}
\end{aligned}
$$

by using the second expression of $Y_{i}(m)^{(u, v)}$ in (3.4). On the other hand, from the equation (3.4), we also obtain

$$
X_{4}(-4)^{(u, v)}=X_{1}(-1)^{(-u,-v)} X_{2}(-2)^{(-u,-v)} X_{3}(-3)^{(-u,-v)} .
$$

By applying this equation on (3.18), we can also be expressed the monomial $M$ as

$$
\begin{aligned}
M= & X_{1}(-1)^{(3,-7)} X_{2}(-1)^{(0,3)} X_{3}(-1)^{(0,0)} X_{4}(-1)^{(0,4)} \\
& \cdot X_{2}(-2)^{(2,-2)} X_{3}(-2)^{(0,2)} X_{4}(-2)^{(0,0)} \\
& \cdot X_{3}(-3)^{(1,-1)} X_{4}(-3)^{(0,1)}
\end{aligned}
$$

Actually, we can obtain this expression of $M$ from (3.6) directly.

Hence we have following marginally large tableau as the image of $M$ under $\Phi^{-1}$.

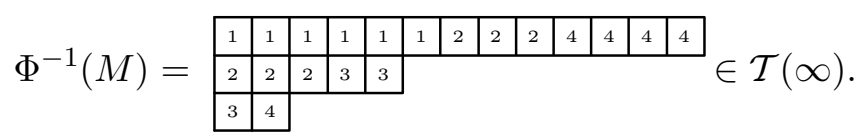




\section{$\S 4$. Monomial description of crystal $\mathcal{B}(\lambda)$}

We introduced a realization of crystal $\mathcal{B}(\lambda)$ given in the monomial language of Kashiwara and Nakajima through Theorem 2.1 and Corollary 2.3. Now, we give an explicit monomial description of $\mathcal{B}(\lambda)$, for the $A_{n}$-case, i.e. we will give a concrete listing of elements belonging to the connected component of $\mathcal{M}_{c}$ (or $\dot{\mathcal{M}}_{c}^{\mathcal{E}}$ ) containing a certain maximal vector $M \in \mathcal{M}_{c}$ (or $\dot{\mathcal{M}}_{c}^{\mathcal{E}}$ ) of weight $\lambda$. Notice that there is a natural identification between crystals $\mathcal{M}_{c}$ and $\dot{\mathcal{M}}_{c}^{\mathcal{E}}$. We will be working on the Nakajima monomial set $\mathcal{M}_{c}$ for convenience.

The organization for this section will follow that of the previous section closely and we take the set $c$ identical to that of (3.1) used for the $\mathcal{B}(\infty)$ case. We fix $\lambda=l_{1} \Lambda_{1}+\cdots+l_{n} \Lambda_{n} \in P^{+}$.

The set we define below was originally obtained by applying Kashiwara actions $\tilde{f}_{i}(i \in I)$ continuously on a maximal vector $\prod_{i \in I} Y_{i}(-i)^{l_{i}} \in \mathcal{M}$ of weight $\lambda$. Actually, we will be able to confirm later that this choice of starting monomial allows us to relate monomials of the set defined below to tableaux in $\mathcal{B}(\lambda)$ naturally.

Definition 4.1. Consider elements of $\mathcal{M}$ having the form

$$
\begin{aligned}
M= & \prod_{i \in I}\left(Y_{i}(-i)^{a_{i}^{i}} \prod_{m=0}^{i-1} Y_{i}(-m)^{a_{i}^{m}}\right) \\
= & Y_{1}(-1)^{a_{1}^{1}} Y_{1}(0)^{a_{1}^{0}} \\
& \cdot Y_{2}(-2)^{a_{2}^{2}} Y_{2}(-1)^{a_{2}^{1}} Y_{2}(0)^{a_{2}^{0}} \\
& \cdots \\
& \cdot Y_{n}(-n)^{a_{n}^{n}} Y_{n}(-n+1)^{a_{n}^{n-1}} \cdots Y_{n}(-1)^{a_{n}^{1}} Y_{n}(0)^{a_{n}^{0}}
\end{aligned}
$$

and satisfying the following conditions

(1) $a_{i}^{i} \geq 0, a_{i}^{0} \leq 0$, and $l_{i}=\sum_{k=0}^{n-i} a_{i+k}^{i}-\sum_{k=0}^{i-1} a_{n-i+1+k}^{k}$ for all $i \in I$,

(2) $\sum_{k=0}^{j} a_{i+k}^{k} \leq 0$ and $\sum_{k=0}^{j} a_{i+k}^{i} \geq 0$ for $1 \leq j \leq n-1,1 \leq i \leq n-j$.

Specifically, in the case of $a_{i}^{j}=0$ for all $i>j$, we have

$$
M=\prod_{i \in I} Y_{i}(-i)^{l_{i}}=Y_{1}(-1)^{l_{1}} Y_{2}(-2)^{l_{2}} \cdots Y_{n}(-n)^{l_{n}} .
$$

We denote by $\mathcal{M}(\lambda)$ the set of all monomials of the form (4.1) and by $M_{\lambda}$ the monomial of $(4.2)$. 
In the $A_{3}$ case, we have

$$
\begin{aligned}
M_{\Lambda_{1}+\Lambda_{2}} & =Y_{1}(-1) Y_{2}(-2), \\
\mathcal{M}\left(\Lambda_{1}+\Lambda_{2}\right) & =\left\{\begin{array}{l}
Y_{1}(-1) Y_{2}(-2), Y_{1}(0)^{-1} Y_{2}(-2) Y_{2}(-1), Y_{1}(-1)^{2} Y_{2}(-1)^{-1}, \\
Y_{2}(-2) Y_{2}(0)^{-1}, Y_{1}(-1) Y_{1}(0)^{-1}, \\
Y_{1}(-1) Y_{2}(-1)^{-1} Y_{2}(0)^{-1}, Y_{1}(0)^{-2} Y_{2}(-1), Y_{1}(0)^{-1} Y_{2}(0)^{-1}
\end{array}\right\} .
\end{aligned}
$$

Remark 4.2. Since this set $\mathcal{M}(\lambda)$ contains a maximal vector $M_{\lambda}$ of weight $\lambda$, by Theorem 2.1 it is enough to prove that the set $\mathcal{M}(\lambda)$ is closed and connected under Kashiwara operators (2.1) and (2.2) on $\mathcal{M}$ to show that $\mathcal{M}(\lambda)$ is a description of the crystal $\mathcal{B}(\lambda)$.

For convenience, we shall introduce new expressions for elements of $\mathcal{M}(\lambda)$ in terms of the new variable

$$
X_{i}(m)=Y_{i}(m) Y_{i-1}(m+1)^{-1} \in \mathcal{M} .
$$

These correspond to $X_{i}(m)^{(0,1)} \in \dot{\mathcal{M}}^{\mathcal{E}}$ introduced in Definition 3.2 under the identification between crystals $\mathcal{M}$ and $\dot{\mathcal{M}}^{\mathcal{E}}$ mentioned in Remark 2.2 .

Proposition 4.3. Consider elements of $\mathcal{M}$ having the form

$$
\begin{aligned}
M= & \prod_{i \in I}\left(X_{i}(-i)^{b_{i}^{i}} \prod_{k=i+1}^{n+1} X_{k}(-i)^{b_{k}^{i}}\right) \\
= & X_{1}(-1)^{b_{1}^{1}} X_{2}(-1)^{b_{2}^{1}} \cdots X_{n}(-1)^{b_{n}^{1}} X_{n+1}(-1)^{b_{n+1}^{1}} \\
& \cdot X_{2}(-2)^{b_{2}^{2}} X_{3}(-2)^{b_{3}^{2}} \cdots X_{n+1}(-2)^{b_{n+1}^{2}} \\
& \cdots \\
& \cdot X_{n}(-n)^{b_{n}^{n}} X_{n+1}(-n)^{b_{n+1}^{n}}
\end{aligned}
$$

satisfying the conditions

(1) $b_{j}^{i} \geq 0$ for all $i, j$,

(2) $\sum_{j=i}^{n+1} b_{j}^{i}=\sum_{k=i}^{n} l_{k}$ for each $i \in I$,

(3) $\sum_{k=0}^{j} b_{i+k}^{i} \geq \sum_{k=0}^{j} b_{i+1+k}^{i+1}$ for $0 \leq j \leq n-1,1 \leq i \leq n-\max \{1, j\}$.

Each element of $\mathcal{M}(\lambda)$ may be written uniquely in this form. Conversely, any element of this form is an element of $\mathcal{M}(\lambda)$. 
Proof. Given any monomial $M=\prod_{i \in I}\left(Y_{i}(-i)^{a_{i}^{i}} \prod_{m=0}^{i-1} Y_{i}(-m)^{a_{i}^{m}}\right) \in$ $\mathcal{M}(\lambda)$, through routine computation, we can obtain the expression

$$
\begin{aligned}
& M=X_{1}(-1)^{\sum_{i=1}^{n} a_{i}^{i}} X_{2}(-1)^{-a_{1}^{0}} X_{3}(-1)^{-a_{2}^{0}} \cdots X_{n+1}(-1)^{-a_{n}^{0}} \\
& \quad \cdot X_{2}(-2)^{\sum_{i=2}^{n} a_{i}^{i}} X_{3}(-2)^{-a_{1}^{0}-a_{2}^{1}} X_{4}(-2)^{-a_{2}^{0}-a_{3}^{1}} \cdots X_{n+1}(-2)^{-a_{n-1}^{0}-a_{n}^{1}} \\
& \quad \cdots \\
& \quad \cdot X_{n-1}(-n+1)^{\sum_{i=n-1}^{n} a_{i}^{i}} X_{n}(-n+1)^{-\sum_{i=0}^{n-2} a_{i+1}^{i}} X_{n+1}(-n+1)^{-\sum_{i=0}^{n-2} a_{i+2}^{i}} \\
& \cdot X_{n}(-n)^{a_{n}^{n}} X_{n+1}(-n)^{-\sum_{i=0}^{n-1} a_{i+1}^{i}} .
\end{aligned}
$$

Since $M \in \mathcal{M}(\lambda)$, from the condition given in (4.1), we obtain the form given in (4.3). The element $M_{\lambda}=\prod_{i \in I} Y_{i}(-i)^{l_{i}}$ can be rewritten in the form $\prod_{i \in I} X_{i}(-i)^{\sum_{k=i}^{n} l_{k}}$.

Conversely, given any monomial of the form (4.3), we have

$$
\begin{aligned}
M= & Y_{1}(-1)^{b_{1}^{1}-b_{2}^{2}} Y_{1}(0)^{-b_{2}^{1}} \\
& \cdot Y_{2}(-2)^{b_{2}^{2}-b_{3}^{3}} Y_{2}(-1)^{b_{2}^{1}-b_{3}^{2}} Y_{2}(0)^{-b_{3}^{1}} \\
& \cdots \\
& \cdot Y_{n-1}(-n+1)^{b_{n-1}^{n-1}-b_{n}^{n}} Y_{n-1}(-n+2)^{b_{n-1}^{n-2}-b_{n}^{n-1}} \cdots Y_{n-1}(-1)^{b_{n-1}^{1}-b_{n}^{2}} Y_{n-1}(0)^{-b_{n}^{1}} \\
& \cdot Y_{n}(-n)^{b_{n}^{n}} Y_{n}(-n+1)^{b_{n}^{n-1}-b_{n+1}^{n}} \cdots Y_{n}(-1)^{b_{n}^{1}-b_{n+1}^{2}} Y_{n}(0)^{-b_{n+1}^{1}} .
\end{aligned}
$$

It is now straightforward to check that $M \in \mathcal{M}(\lambda)$. We have thus shown that $\mathcal{M}(\lambda)$ consists of elements of the form (4.3).

Using this new expression for $\mathcal{M}(\lambda)$, we can easily obtain the following fact.

Corollary 4.4. For $\mu, \tau \in P^{+}, \mathcal{M}(\mu+\tau)=\mathcal{M}(\mu) \mathcal{M}(\tau)$, where $\mathcal{M}(\mu) \mathcal{M}(\tau)=\left\{M \cdot M^{\prime} \mid M \in \mathcal{M}(\mu), M^{\prime} \in \mathcal{M}(\tau)\right\}$.

Now, we translate the Kashiwara operator actions (2.1), (2.2) into a form suitable for the new monomial expression of $\mathcal{M}(\lambda)$. In a manner similar to the proofs of Lemmas 3.6 and 3.7 for the $\mathcal{M}(\infty)$ case, we obtain the following result.

LEMMA 4.5. The set $\mathcal{M}(\lambda)$ is closed under the action given below: Fix element

$$
M=\prod_{i \in I}\left(X_{i}(-i)^{b_{i}^{i}} \prod_{k=i+1}^{n+1} X_{k}(-i)^{b_{k}^{i}}\right) \in \mathcal{M}(\lambda) .
$$


Consider the following finite ordered sequence of components of $M$

$$
\begin{gathered}
X_{n+1}(-1)^{b_{n+1}^{1}}, X_{n}(-1)^{b_{n}^{1}}, \ldots, X_{1}(-1)^{b_{1}^{1}}, \\
X_{n+1}(-2)^{b_{n+1}^{2}}, X_{n}(-2)^{b_{n}^{2}}, \ldots, X_{2}(-2)^{b_{2}^{2}}, \\
\ldots, \\
X_{n+1}(-n)^{b_{n+1}^{n}}, X_{n}(-n)^{b_{n}^{n}} .
\end{gathered}
$$

(1) For $i \in I$, under each component $X_{i+1}(-m)^{b_{i+1}^{m}}$ from the above sequence, write $b_{i+1}^{m}$-many 1 's, and under each $X_{i}(-m)^{b_{i}^{m}}$, write $b_{i}^{m}-$ many 0's.

(2) From this sequence of 1's and 0 's, successively cancel out each $(0,1)$ pair to obtain a sequence of 1's followed by 0 's (reading from left to right). This remaining sequence of 1 's and 0 's is called the $i$-signature of $M$.

(3) We define

$$
\tilde{f}_{i} M=M X_{i}(-m)^{-1} X_{i+1}(-m)=M A_{i}(-m)^{-1}
$$

if $X_{i}(-m)^{b_{i}^{m}}$ is the component corresponding to the left-most 0 appearing in the i-signature of $M$. And we define

$$
\tilde{e}_{i} M=M X_{i}(-m) X_{i+1}(-m)^{-1}=M A_{i}(-m)
$$

if $X_{i+1}(-m)^{b_{i+1}^{m}}$ corresponds to the right-most 1 .

(4) We define $\tilde{e}_{i} M=0$ if no 1 remains and $\tilde{f}_{i} M=0$ if no 0 remains.

Lemma 4.6. The operation given in Lemma 4.5 is just another expression of the Kashiwara operators given on $\mathcal{M}$, restricted to $\mathcal{M}(\lambda)$.

From the above lemmas, we obtain the following result immediately.

Proposition 4.7. The set $\mathcal{M}(\lambda)$ forms a $U_{q}\left(A_{n}\right)$-subcrystal of $\mathcal{M}$.

Figures 5 and 6 are the crystal graphs of $\mathcal{M}\left(\Lambda_{1}+\Lambda_{2}\right)$ for type $A_{2}$.

Now, we will show that $\mathcal{M}(\lambda)$ is a new description of $\mathcal{B}(\lambda)$. Recall that we identify elements of the highest weight crystal $\mathcal{B}(\lambda)$ with semistandard tableaux of $\lambda$-shape, for the $A_{n}$ case. We define a canonical map $\Psi: \mathcal{B}(\lambda) \rightarrow$ 


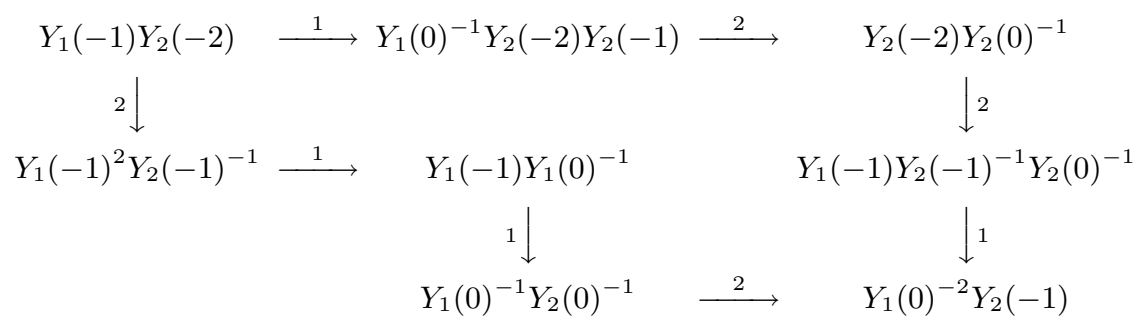

Figure 5: The crystal graph $\mathcal{M}\left(\Lambda_{1}+\Lambda_{2}\right)$ for type $A_{2}$

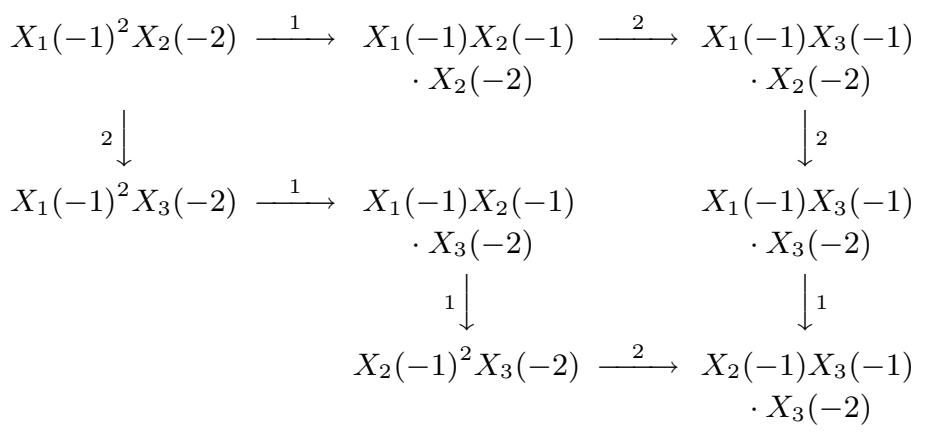

Figure 6: The crystal $\mathcal{M}\left(\Lambda_{1}+\Lambda_{2}\right)$ for type $A_{2}$

$\mathcal{M}(\lambda)$ by setting $\Psi(S)=M$, where $S$ is the semistandard tableau of shape $\lambda$ with each $i$ th row made up of $b_{k}^{i}$-many $k$-blocks for $i \leq k \leq n+1$ and

$$
M=\prod_{i \in I}\left(X_{i}(-i)^{b_{i}^{i}} \prod_{k=i+1}^{n+1} X_{k}(-i)^{b_{k}^{i}}\right) \in \mathcal{M}(\lambda) .
$$

It is obvious that this map $\Psi$ is well-defined and that it is actually bijective.

The action of Kashiwara operators on the new expressions for elements of $\mathcal{M}(\lambda)$ given in Lemma 4.5 follows the process for defining it on semistandard tableaux of $\mathcal{B}(\lambda)$. Hence the map $\Psi$ naturally commutes with the Kashiwara operators $\tilde{f}_{i}$ and $\tilde{e}_{i}$. Other parts of the proof showing that $\Psi$ is a crystal isomorphism are easy.

THEOREM 4.8. The bijection $\Psi$ is a $U_{q}\left(A_{n}\right)$-crystal isomorphism. Therefore, we have a $U_{q}\left(A_{n}\right)$-crystal isomorphism

$$
\mathcal{B}(\lambda) \stackrel{\sim}{\longrightarrow} \mathcal{M}(\lambda) .
$$


Remark 4.9. As we mentioned in Remark 4.2, to prove that $\mathcal{M}(\lambda)$ is a description of the crystal $\mathcal{B}(\lambda)$, it is enough to show that the set $\mathcal{M}(\lambda)$ is closed and connected under Kashiwara operators (2.1) and (2.2) on $\mathcal{M}$. In Proposition 4.7, the closedness was shown, so now it remains to show the connectedness. But, in the above theorem, we proved that $\mathcal{M}(\lambda)$ is isomorphic to $\mathcal{B}(\lambda)$ in a direct manner.

Now we have an explicit monomial description of $\mathcal{B}(\lambda)$, which is a connected component in $\mathcal{M}$ of the maximal vector $M_{\lambda}$ of weight $\lambda$.

We can obtain more general results as with the $\mathcal{M}(\infty)$ case.

For any $r \in \mathbf{Z}$, set $\mathcal{M}(r ; \lambda)$ to be the set of all elements in $\mathcal{M}$ of the form

$$
\prod_{i \in I}\left(Y_{i}(r-i)^{a_{i}^{i}} \prod_{m=0}^{i-1} Y_{i}(r-m)^{a_{i}^{m}}\right)
$$

satisfying the same condition given to (4.1). Specifically, in the case of $a_{i}^{j}=0$ for all $i>j$, we set

$$
M_{(r ; \lambda)}=\prod_{i \in I} Y_{i}(r-i)^{l_{i}}
$$

Each element of the set $\mathcal{M}(r ; \lambda)$ may be written in the form

$$
M=\prod_{i \in I}\left(X_{i}(r-i)^{b_{i}^{i}} \prod_{k=i+1}^{n+1} X_{k}(r-i)^{b_{k}^{i}}\right)
$$

satisfying the same conditions given to (4.3). Conversely, any element of this form is an element of $\mathcal{M}(r ; \lambda)$.

The set $\mathcal{M}(\lambda)$ is a special case of this set $\mathcal{M}(r ; \lambda)$ which is $r=0$.

Through a process similar to that given in Propositions 4.3 and 4.7 for $\mathcal{M}(\lambda)$, we can obtain the result that the set $\mathcal{M}(r ; \lambda)$ becomes a crystal which is isomorphic to $\mathcal{M}(\lambda)$. The isomorphism maps $M_{\lambda}$ of $\mathcal{M}(\lambda)$ onto the vector $M_{(r ; \lambda)}$. Thus, the crystal $\mathcal{M}(r ; \lambda)$ is also a description of $\mathcal{B}(\lambda)$. Namely, we gave a concrete listing of elements belonging to the connected component $\mathcal{M}(r ; \lambda)$ of $\mathcal{M}$ containing a maximal vector $M_{(r ; \lambda)} \in \mathcal{M}$ of weight $\lambda$.

Finally, we can easily obtain the following fact using the expression (4.6) for $\mathcal{M}(r ; \lambda)$.

Corollary 4.10. For $\mu, \tau \in P^{+}, \mathcal{M}(r ; \mu+\tau)=\mathcal{M}(r ; \mu) \mathcal{M}(r ; \tau)$. 


\section{REFERENCES}

[1] G. Cliff, Crystal bases and Young tableaux, J. Algebra, 202 (1998), no. 1, 10-35.

[2] J. Hong and S.-J. Kang, Introduction to quantum groups and crystal bases, Graduate Studies in Mathematics, vol. 42, Amer. Math. Soc., Providence, RI, 2002.

[3] J. Hong and H. Lee, Young tableaux and crystal $\mathcal{B}(\infty)$ for finite simple Lie algebras, arXiv:math.QA/0507448.

[4] J. C. Jantzen, Lectures on quantum groups, Graduate Studies in Mathematics, vol. 6, Amer. Math. Soc., Providence, RI, 1996.

[5] S.-J. Kang, M. Kashiwara, and K. C. Misra, Crystal bases of Verma modules for quantum affine Lie algebras, Compositio Math., 92 (1994), no. 3, 299-325.

[6] S.-J. Kang, J.-A. Kim, and D.-U. Shin, Monomial realization of crystal bases for special linear Lie algebras, J. Algebra, 274 (2004), no. 2, 629-642.

[7] S.-J. Kang, J.-A. Kim, and D.-U. Shin, Crystal bases for quantum classical algebras and Nakajima's monomials, Publ. Res. Inst. Math. Sci., 40 (2004), no. 3, 757-791.

[8] M. Kashiwara, The crystal base and Littelmann's refined Demazure character formula, Duke Math. J., 71 (1993), no. 3, 839-858.

[9] M. Kashiwara, Realizations of crystals, Combinatorial and geometric representation theory (Seoul, 2001), Contemp. Math., vol. 325, Amer. Math. Soc., Providence, RI, 2003, pp. 133-139.

[10] M. Kashiwara and T. Nakashima, Crystal graphs for representations of the q-analogue of classical Lie algebras, J. Algebra, 165 (1994), no. 2, 295-345.

[11] M. Kashiwara and Y. Saito, Geometric constructin of crystal bases, Duke Math. J., 89 (1997), 9-36.

[12] J.-A. Kim, Monomial realization of crystal graphs for $U_{q}\left(A_{n}^{(1)}\right)$, Math. Ann., 332 (2005), no. 1, 17-35.

[13] H. Lee, Extended Nakajima monomials and realization of crystal $\mathcal{B}(\infty)$, KIAS preprint M05008.

[14] H. Lee, Realizations of crystal $\mathcal{B}(\infty)$ using Young tableaux and Young walls, J. Algebra, in press.

[15] H. Lee, Extended Nakajima monomials and crystal $\mathcal{B}(\infty)$ for finite simple Lie algebras, KIAS preprint M05019, J. Algebra, in press.

[16] H. Nakajima, t-analogue of the q-characters of finite dimensional representations of quantum affine algebras, Physics and Combinatorics (Nagoya, 2000), World Sci. Publ., River Edge, NJ, 2001, pp. 196-219.

[17] H. Nakajima, t-analogs of q-characters of quantum affine algebras of type $A_{n}, D_{n}$, Combinatorial and geometric representation theory (Seoul, 2001), Contemp. Math., vol. 325, Amer. Math. Soc., Providence, RI, 2003, pp. 141-160.

[18] T. Nakashima and A. Zelevinsky, Polyhedral realizations of crystal bases for quantized Kac-Moody algebras, Adv. Math., 131 (1997), no. 1, 253-278.

[19] Y. Saito, Combinatorial and geometric realization of crystal $\mathcal{B}(\infty)$ for type $A_{n}$, KIAS lecture, 2002.

[20] A. Savage, A geometric construction of crystal graphs using quiver varieties: extension to the non-simply laced case, Infinite-dimensional aspects of representation 
theory and applications, Contemp. Math., vol. 392, Amer. Math. Soc., Providence, RI, 2005, pp. 133-154.

[21] A. Savage, Geometric and combinatorial realizations of crystals of enveloping algebras, arXiv:math.QA/0601511, Contemp. Math., in press.

[22] D.-U. Shin, Crystal bases and monomials for $U_{q}\left(G_{2}\right)$-modules, KIAS preprint M04012.

Korea Institute for Advanced Study

207-43 Cheongryangri-dong

Dongdaemun-gu

Seoul 130-722

Korea

hmlee@kias.re.kr 